\title{
L'union fait la force
}

Le verdict est tombé le $1^{\text {er }}$ juin à 15.26 h: le week-end dernier, le peuple suisse a clairement rejeté par 69,5\% des voix l'article constitutionnel proposé et donc la prétention des caisses aux pleins pouvoirs. Et les citoyens suisses ont aussi dit oui sans ambiguïté au libre choix du médecin!

Le corps médical s'est lancé dans la bataille il y a quelques mois: l'Assemblée des délégués a suivi la recommandation du Comité central de la FMH en optant pour un rôle actif de la FMH dans la campagne contre l'article constitutionnel sur la santé. Dès le départ, il était clair que la FMH n'allait pas mener seule ce combat, mais qu'elle s'allierait à d'autres organisations de fournisseurs de prestations pour former des coalitions - c'était une occasion unique! Le comité «NON au diktat des caisses» a ainsi vu le jour et, avec lui, une force d'opposition largement représentative et crédible: médecins, organisations de défense des patients et des consommateurs, pharmaciens, dentistes, infirmières, sages-femmes, physiothérapeutes ont fait front commun contre cet article constitutionnel. En collaboration étroite avec les cantons, c'est-à-dire les directions cantonales de la santé, et avec les partis politiques, il a ainsi été possible d'agir sur l'opinion publique et de la convaincre peu à peu.

En votre qualité de médecin, vous avez fourni une contribution essentielle, sinon la plus importante, à cette campagne de vote! J'aimerais vous en remercier cordialement au nom de la FMH et du comité «NON au diktat des caisses»! Au moyen de prospectus, d'affiches et de pin's, vous avez attiré l'attention de vos patients sur les conséquences négatives de l'article proposé. Nombre d'entre vous sont devenus de véritables militants, ont fourni des témoignages, écrit des lettres de lecteurs, adhéré à des comités cantonaux ou participé à des stands. De nombreuses organisations de médecins se sont encore engagées dans des activités supplémentaires. Un tout grand merci également à toutes les personnes qui ont soutenu notre campagne par des dons!

Lorsque j'ai repris la responsabilité de la communication de la FMH en août 2007, je savais - en théorie - combien le corps médical était hétérogène. Au cours de mon activité, je me suis rendu compte qu'en raison des intérêts particuliers qu'elle regroupe et des structures fédéralistes qui sont les siennes, il ne serait pas aisé de donner à la FMH (qui compte plus de 30000 membres) le profil d'une organisation faîtière forte et unie. Sans oublier qu'un projet visant à renforcer son image avait été rejeté par la Chambre médicale de mai 2007 pour des raisons diverses et compréhensibles.

Cette page-là est maintenant elle aussi tournée, car la campagne de vote a fourni sans conteste une contribution essentielle à la création d'une image de marque de la FMH. Et ce d'une part vers l'extérieur, car la fédération des médecins est entrée en dialogue aussi bien avec le monde politique qu'avec celui des médias, et donc avec le grand public. Il nous faut maintenant prendre soin du réseau mis en place et le développer afin de pouvoir exprimer nos idées et défendre nos objectifs dans les endroits stratégiques. Mais d'autre part, la campagne de vote a également renforcé la FMH en son sein: le corps médical - médecins de premier recours et spécialistes, médecins praticiens et médecins hospitaliers, représentants de toutes les régions de Suisse - a rarement présenté une image aussi unie. La force d'impact ainsi gagnée a déjà exercé ses effets dans la votation qui vient de se dérouler. Conserver cette combativité pour continuer à jouer le rôle d'un acteur-clé dans le système de santé suisse: tel doit être l'objectif du corps médical, aujourd'hui comme à l'avenir.

Jacqueline Wettstein, responsable de la communication de la FMH 